\title{
Aligned Porous Materials by Directional Freezing of Solutions in Liquid $\mathrm{CO}_{2}$ Haifei Zhang, James Long and Andrew I. Cooper* \\ University of Liverpool, Department of Chemistry, Crown Street, Liverpool L69 7ZD, UK \\ *E-mail: aicooper@liv.ac.uk
}

Supporting Information (7 pages, Figures S1-S5) 
Materials All materials were purchased from Aldrich and were used as received without further purification. The stainless steel tubing used for directional freezing was supplied by Supelco(20527, OD $\times$ ID $=1 / 4 \mathrm{in}$. $(6.35 \mathrm{~mm}) \times 0.209$ in. $(5.3 \mathrm{~mm})$. The monolithic composite material shown in Fig. 2b was produced in a larger column $(\mathrm{ID}=20 \mathrm{~mm})$. 


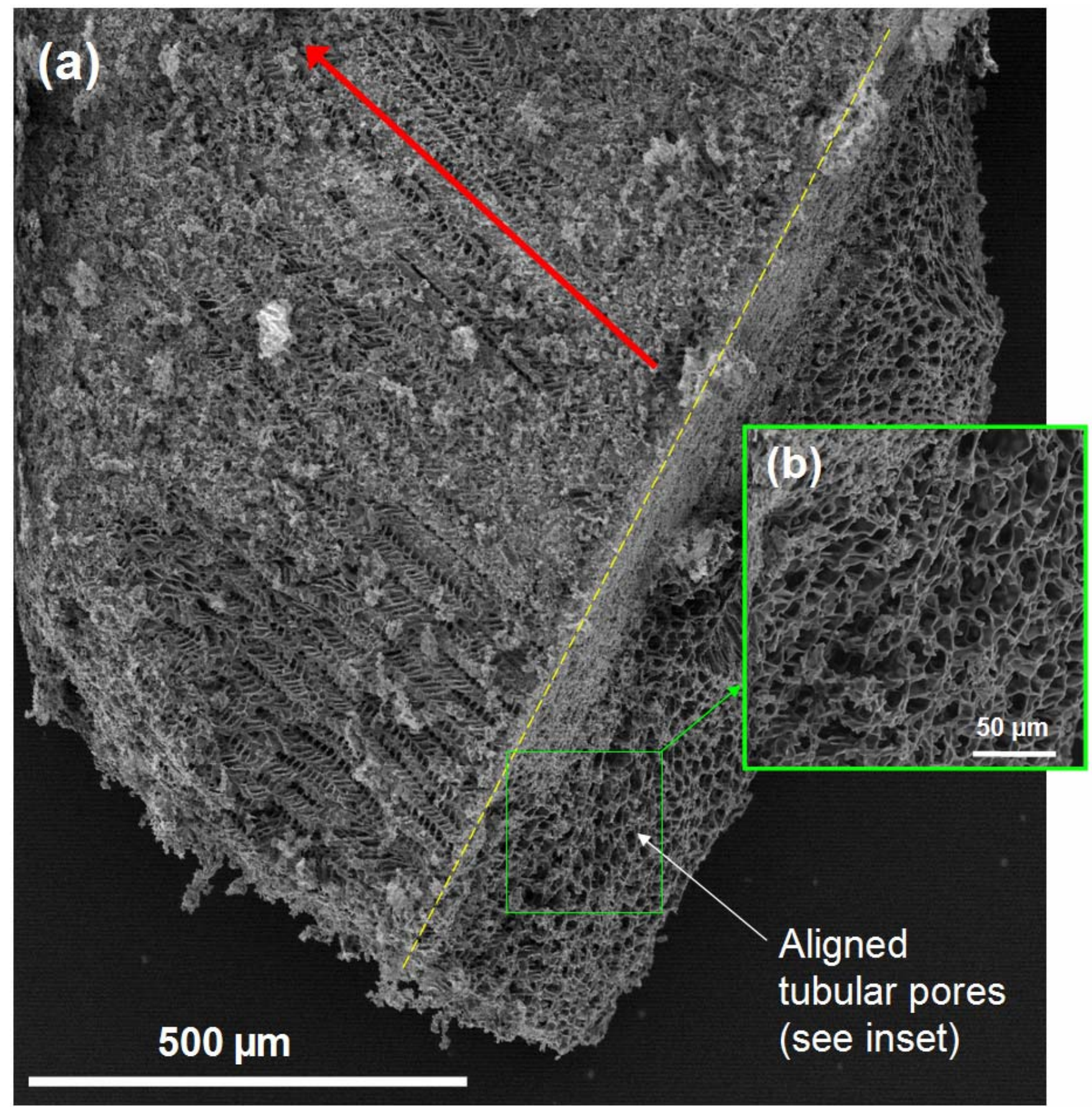

Figure S1: (a) Low magnification electron micrograph of the aligned BGAL material shown in Fig. 2 . The red arrow corresponds to the approximate direction of alignment. The sample has been sectioned both parallel to and perpendicular to the direction of alignment. The dashed yellow line marks the intersection between these two planes. (b) Higher magnification expansion of the tubular pore structure sectioned perpendicular to the direction of alignment. 

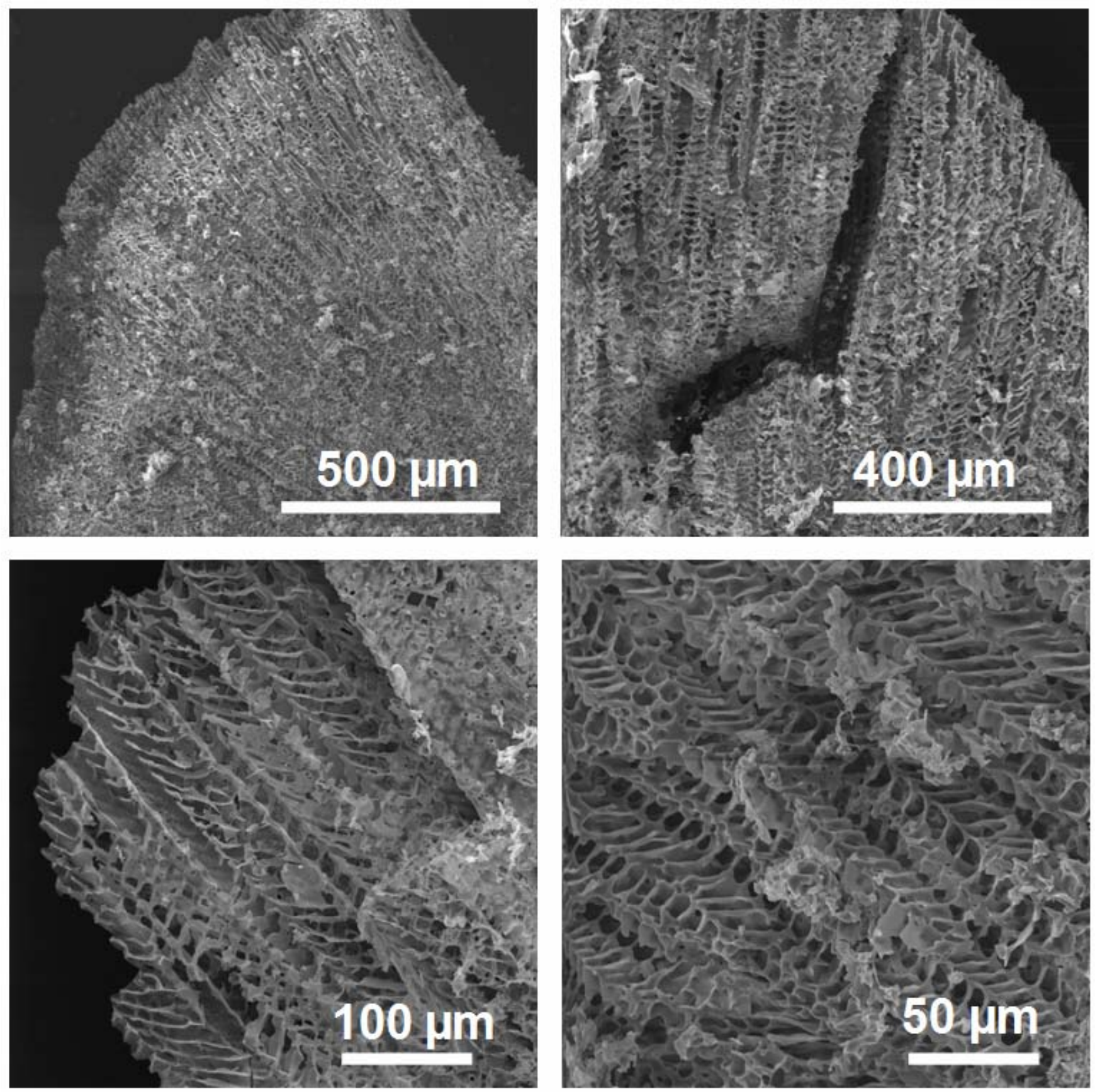

Figure S2: Electron micrographs of showing various sections of the aligned BGAL material shown in Fig. 2. 


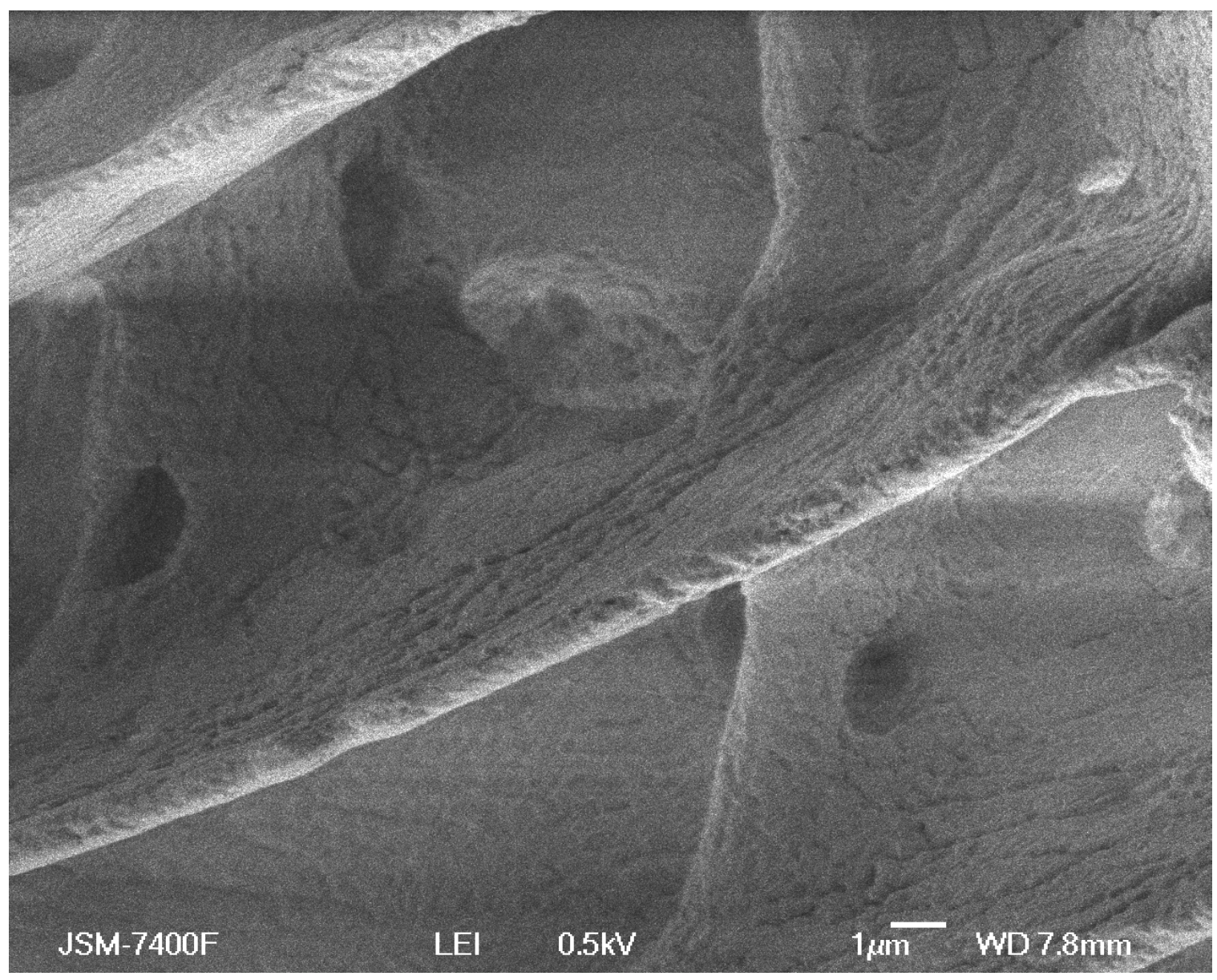

Figure S3: Electron micrograph (FE SEM, no gold coating) showing the fine structure and surface topology of the aligned BGAL material shown in Fig. 2. 


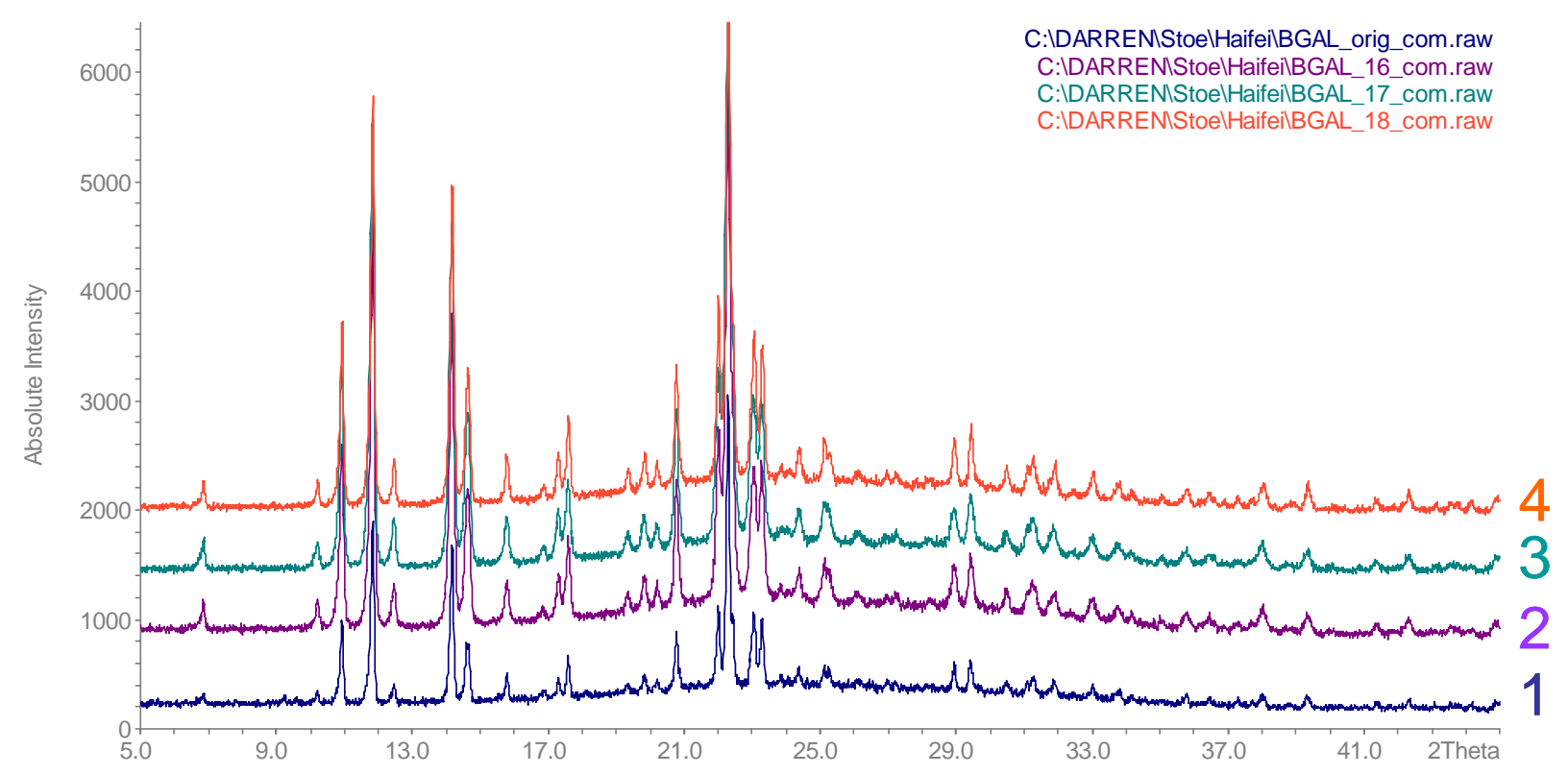

Figure S4: X-ray diffraction data for unprocessed BGAL (1) and three aligned samples processed from $\mathrm{CO}_{2}$ (0.22 g BGAL was dissolved in liquid $\mathrm{CO}_{2}$ in the $1.85 \mathrm{~cm}^{3}$ stainless-steel tubing at $21^{\circ} \mathrm{C}$ and different pressures, 60 bar (2), 100 bar (3), and 150 bar (4)). 

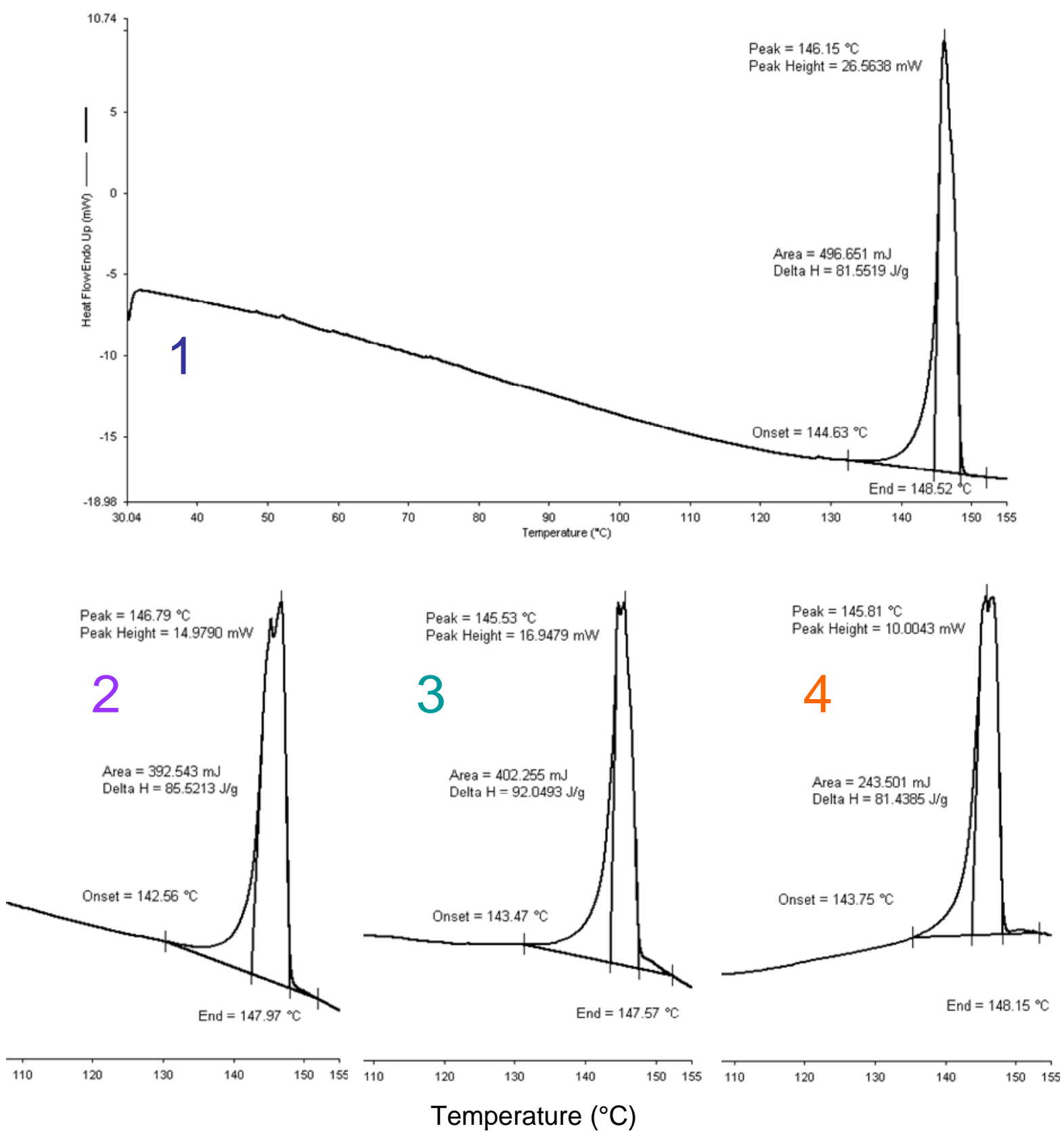

Figure S5: Thermal analysis for unprocessed BGAL (1) and three aligned samples processed from $\mathrm{CO}_{2}(0.22 \mathrm{~g}$ BGAL was dissolved in liquid $\mathrm{CO}_{2}$ in the $1.85 \mathrm{~cm}^{3}$ stainless-steel tubing at $21^{\circ} \mathrm{C}$ and different pressures, 60 bar (2), 100 bar (3), and 150 bar (4)). 